\title{
Dielectric and Electric Modulus Behavior of Chlorinated Poly(Vinyl Chloride) Stabilized with Phenyl Maleimide
}

\author{
Taha A. Hanafy ${ }^{1,2}$ \\ ${ }^{1}$ Department of Physics, Faculty of Science, Tabuk University, Tabuk, KSA \\ ${ }^{2}$ Physics Department, Faculty of Science, Fayoum University, El Fayoum, Egypt \\ Email: tahanafy2@yahoo.com
}

Received October 5, 2012; revised November 8, 2012; accepted November 15, 2012

\begin{abstract}
Dielectric constant, $\varepsilon^{\prime}$, dielectric loss factor, $\varepsilon^{\prime \prime}$, electric modulus, $M$, and ac conductivity, $\sigma_{\mathrm{ac}}$, of pure CPVC and that stabilized with $10 \mathrm{wt} \%$ of phenyl maleimide, PM, have been carried out. The dielectric properties have been studied in the temperature and frequency ranges; $310 \mathrm{~K}-450 \mathrm{~K}$ and $1 \mathrm{kHz}-4 \mathrm{MHz}$, respectively. The incorporation of $10 \mathrm{wt} \%$ of $\mathrm{PM}$ as stabilizer for CPVC leads to reduce its $T g$ from $405 \mathrm{~K}$ to $378 \mathrm{~K}$ at $10 \mathrm{kHz}$. PM molecules within CPVC structure reduce the double bond, stabilizer effect, and cause the widely spacing between CPVC main chains, plasticizer effect. Three dielectric relaxation processes namely $\rho, \alpha^{\prime}$, and $\alpha$ were observed for pure CPVC. The first process was explained based on space charge formation or Maxwell-Wagner-Sillers, MWS, polarization. The second one is due to the segmental motion of the branching of CPVC. The third process occurs around the glass-rubber temperature, $T g$, and is related to the micro-Brownian motion of the main polymer chain. Electric modulus and ac conductivity reveal that the conduction mechanism of CPVC is follow the correlated barrier hopping, $\mathrm{CBH}$, while stabilized sample exhibits a quantum mechanical tunneling, QMT, type conduction.
\end{abstract}

Keywords: CPVC; Stabilizer; Plasticizer; Dielectric Relaxation; Hopping Conduction

\section{Introduction}

Chlorinated polyvinyl chloride, CPVC, is produced by post-chlorination of polyvinyl chloride, PVC. CPVC has high heat distortion temperature, chemical inertness, and outstanding mechanical. It has good flame retardant and smoke density properties [1-5]. Chlorination of PVC enhances the mechanical properties of the resulting polymer. Chlorination of $\mathrm{PVC}$ increases the glass rubber transition temperature, $\mathrm{Tg}$, [4] from $80^{\circ} \mathrm{C}$ to $90^{\circ} \mathrm{C}$ and makes the materials suitable for use in high temperature applications [6]. Both PVC and CPVC can not be processed without the addition of stabilizer and plasticizer. Commercial CPVC stabilizers are usually either basic lead salt $[7,8]$, which can trap the evolved $\mathrm{HCl}$ gas, thus delaying the polymer degradation or metallic soap esters [9]. The stabilizing action occurs through displacement of the labile chlorine atoms on the polymer chains by the ester from the decomposed stabilizer. Recently, it was reported that the incorporation of organic materials like $\mathrm{N}$-phenyl maleimides as a stabilizers for PVC and CPVC $[10,11]$. Organic stabilizers have the advantage of being metal free and environmentally acceptable. N-phenyl maleimides are known have high thermal stability $[9,10]$. These stabilizers trap the radical species in the degradation process by blocking the newly formed radical sites on the polymeric chains and absorption of liberated $\mathrm{HCl}$ gas.

Dielectric relaxation spectroscopy, DRS, of polymers provides information about the orientation and translational adjustment of mobile charge present in the dielectric medium. The energy transferred to the dielectric material is a function not only of the applied electric field but also depends on the physical properties of the material. The dependence of contributions of different components of dielectric polarization, such as electric, ionic, and orientational polarization on the frequency of the applied electric field, is responsible for the change in the value of the dielectric constant. The relative permittivity, $\varepsilon^{*}(\omega)$, of a dielectric as a function of the frequency is given by [12]:

$$
\varepsilon^{*}=\varepsilon^{\prime}(\omega)-i \varepsilon^{\prime \prime}(\omega)
$$

where $\varepsilon^{\prime}(\omega)$ is the dielectric constant characterizes the most important property of the dielectric material. The dielectric loss index, $\varepsilon^{\prime \prime}(\omega)$, characterizes the dissipation of energy of the electric oscillation within the dielectric medium. The magnitude of $\varepsilon^{\prime}(\omega)$ of the polymeric material is determined by its chemical constitution, structure, and composition. The parameters that characterize the dielectric loss index and the dielectric loss tangent, $\tan (\delta)$, 
depend upon the specific features of molecular main chain motion within the polymer. Real dielectric polymers are commonly described by a spectrum of relaxation times. The relaxation spectra appear due to presence of long polymeric chain and specific inter-molecular interactions. The simplest form of $\varepsilon^{\prime}(\omega)$ and $\varepsilon^{\prime \prime}(\omega)$ for the relaxation process that characterized by a single relaxation time $(\tau)$ is given by[12]:

$$
\begin{gathered}
\varepsilon^{\prime}(\omega)=\varepsilon^{\prime}(\infty)+\frac{\Delta \varepsilon}{1+\omega^{2} \tau^{2}} \\
\mathcal{E}^{\prime \prime}(\omega)=\frac{\Delta \varepsilon \omega \tau}{1+\omega^{2} \tau^{2}}
\end{gathered}
$$

where $\Delta \varepsilon=\varepsilon^{\prime}(o)-\varepsilon^{\prime}(\infty)$ is the dielectric strength, $\varepsilon^{\prime}(o)$, and $\varepsilon^{\prime}(\infty)$ are the relaxed and unrelaxed dielectric constant, respectively.

Different experimental techniques, including differential scanning calorimetry, DSC, dynamic mechanical thermal analysis, DMTA, and dielectric relaxation spectroscopy, DRS, are used for the determination of glass rubber transition, $T g$ of the polymeric materials [13]. In this work the temperature and frequency dependence of $\varepsilon^{\prime \prime}$ is used to determine $T g$ of the investigated samples, where the $\alpha$-relaxation process is accompanied with the $\mathrm{Tg}$ of the polymeric material. Also, we investigate the effect of an additive phenyl maleimide to improve the performance of CPVC. Also, the dielectric properties, electric modulus, and ac conductivity of pure CPVC and that stabilized with $10 \mathrm{wt} \%$ of PM were investigated to shed light on their molecular relaxation and conduction mechanism.

\section{Experimental}

CPVC used in the present work was supplied by Weihai Jinhong Chemicals Ltd. with chlorine content $69 \%$ and density $1.4 \mathrm{~g} / \mathrm{cm}^{3}$. Phenyl maleimide was prepared by the method described by Searle $[10,14]$. CPVC film stabilized with $10 \mathrm{wt} \%$ of PM was prepared by casting as follows: CPVC was dissolved in tetrahydrofuran (THF, Aldrich) at $50^{\circ} \mathrm{C}$ for $30 \mathrm{~min}$ with continuous stirring. A $10 \mathrm{wt} \%$ of PM was also dissolved in THF at $50^{\circ} \mathrm{C}$ and added to CPVC solution. The mixture was left $8 \mathrm{~h}$, with continuous stirring, to reach a suitable viscosity. The solution of the mixture was cast into a Petri dish placed on a leveled plate at $30^{\circ} \mathrm{C}$ for 10 days until the solvent was completely evaporated. The obtained film of $0.1 \mathrm{~mm}$ thickness was cut into square pieces and then coated with silver paste to achieve ohmic contacts.

The dielectric measurements were carried out with a Hioko 3532 RLC bridge (Nagano, Japan) in the frequency and temperature ranges of $50 \mathrm{kHz}$ to $1 \mathrm{MHz}$ and $300-450 \mathrm{~K}$, respectively. The dielectric constant, dielectric loss index, and alternating current conductiv- ity, $\sigma_{\mathrm{ac}}$, were calculated by [5]:

$$
\begin{gathered}
\mathcal{E}^{\prime}=\frac{C d}{\varepsilon_{o} l} \\
\mathcal{E}^{\prime \prime}=\varepsilon^{\prime} \tan (\delta) \\
\sigma_{\mathrm{ac}}=\omega \varepsilon_{o} \varepsilon^{\prime \prime}
\end{gathered}
$$

where $C$ is the capacitance of the sample-filled capacitor, $d$ is the sample thickness, $\varepsilon_{0}$ is the permittivity of the free space, $l$ is the electrode area, and $\omega$ is the angular frequency. The temperature of the sample was measured with a T-type thermocouple. The measurement accuracy for the temperature was about \pm 1 " $\mathrm{K}$ ".

\section{Results and Discussion}

\subsection{Dielectric Formalism}

The frequency dependence of $\varepsilon^{\prime}$ for pure CPVC and that stabilized with PM at some fixed temperatures is shown in Figures 1(a) and (b). It is clear that the values of $\varepsilon^{\prime}$ decrease with increasing the frequency due to decreasing number of dipoles which contribute to polarization. At very low frequency $(\omega \ll 1 / \tau)$, where $\tau$ is the relaxation time, the dipoles can follow the field and $\varepsilon^{\prime} \approx \varepsilon_{0}$. As the frequency increases $(\omega<1 / \tau)$, the dipoles begin to lag the field and $\varepsilon^{\prime}$ slightly decreases. When the frequency reaches a characteristic value $(\omega=1 / \tau)$, the dielectric constant exhibits relaxation process [15]. The dielectric constant of the stabilized CPVC sample increases slightly compared to that of pure one. This can be discussed as follows: the stabilized molecules are compatible with CPVC and are distributed inter-structurally within the free volume of the non dense globular areas of CPVC [5,16]. This will increase the segmental mobility of the non array macromolecules and passage chains [17]. In addition, with the increase of temperature the thermo-mechanical motion of PM molecules become significance and cooperative nature. Also, PM molecules can form channel cluster in the polymeric glassy state $[18,19]$. These clusters have many polar groups $(\mathrm{C}=\mathrm{O}$ and phenyl groups $)$ and began to move at high temperature in cooperation with $\mathrm{C}-\mathrm{Cl}$ dipolar groups of CPVC. This will increase $\varepsilon^{\prime}$ of the stabilized CPVC sample.

Figures 2(a) and (b) represent the frequency dependence of $\varepsilon^{\prime \prime}$ for pure CPVC and that stabilized with 10 $\mathrm{wt} \%$ of $\mathrm{PM}$ at some fixed temperatures. From Figure 2 (a), $\varepsilon^{\prime \prime}$ of pure CPVC undergoes three relaxation processes namely, $\rho$-, $\alpha^{\prime}$-, and $\alpha$-peaks. The first is located at $10 \mathrm{kHz}$. It originates from the motion of space charges that are accumulated on the polymeric sample close to electrode [20]. The nature of $\rho$-relaxation process for crystalline regions is due to the chain trapping of interfaces or Maxwell-Wagner-Sillers, MWS, polarization [21]. 


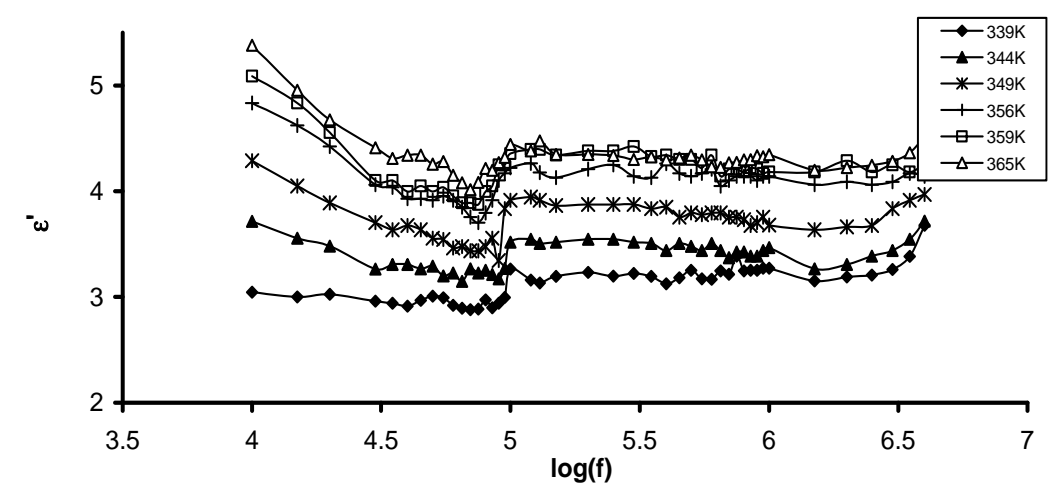

(a)

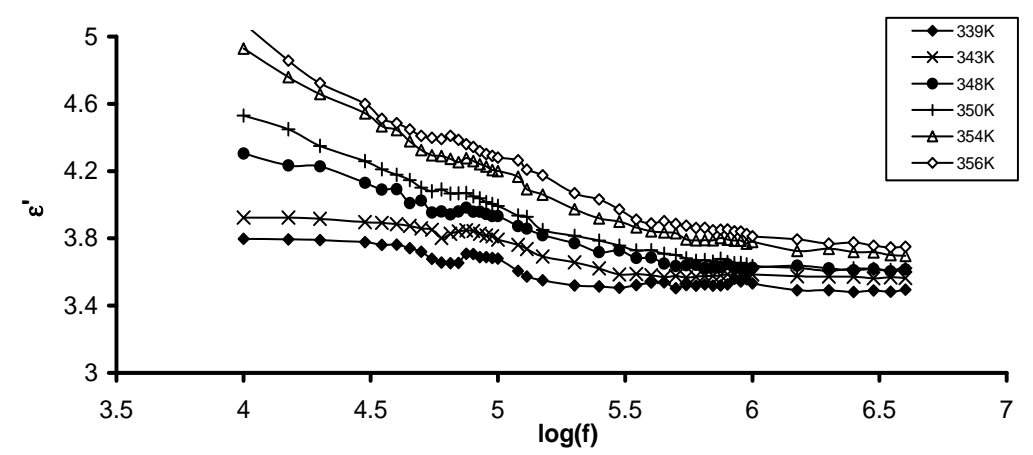

(b)

Figure 1. Frequency dependence of $\varepsilon^{\prime}$ (a) Pure CPVC and (b) CPVC stabilized with 10 wt\% of PM at some constant temperatures.

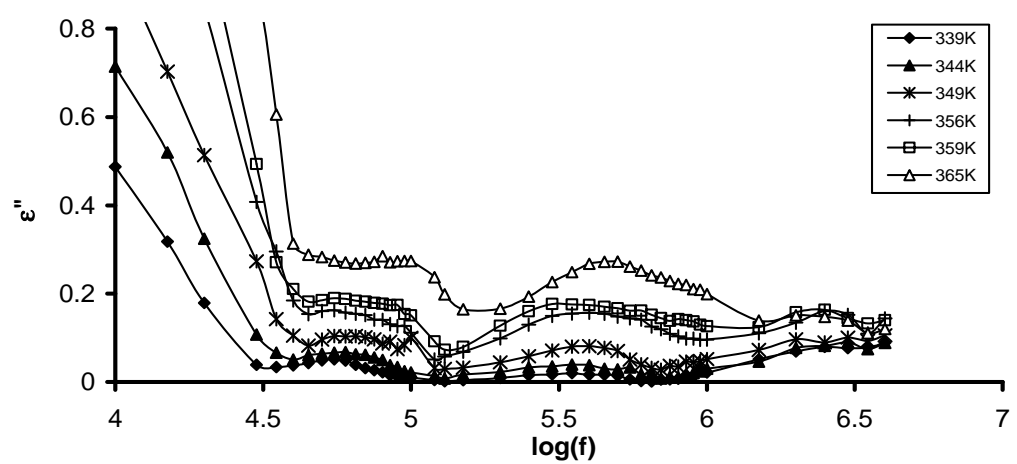

(a)

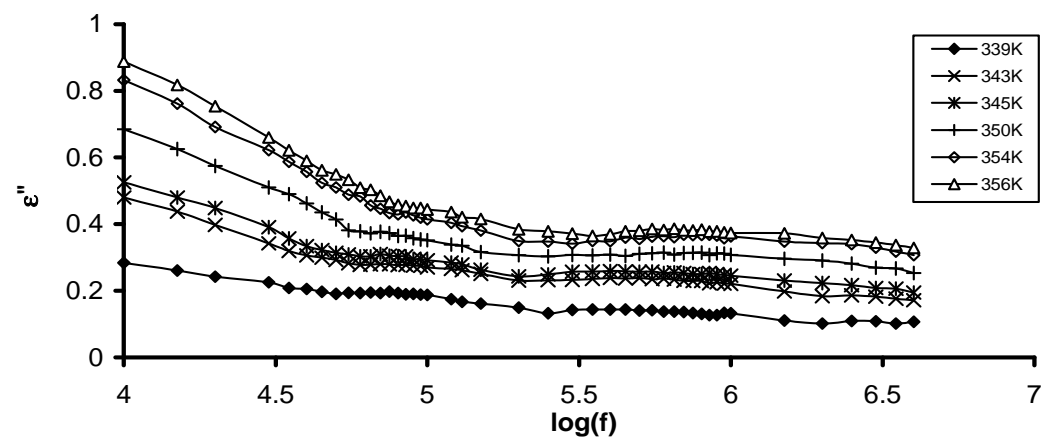

(b)

Figure 2. Frequency dependence of $\varepsilon^{\prime \prime}$ (a) Pure CPVC and (b) CPVC stabilized with 10 wt\% of PM at some constant temperatures. 
However, in the amorphous phases, $\rho$-process is related to conductive impurities, injected space change and electrode polarizations. In semi-crystalline polymer such as PVC and CPVC, chain trapping or MWS polarization is more acceptable [20]. For stabilized CPVC sample, $\rho$-relaxation can be related to the multiple phases which come from the channel cluster formation within the sample $[18,19]$. The second process, $\alpha^{\prime}$-relaxation, is observed at $80 \mathrm{kHz}$. The process has been reported for PVC $[17,21]$ but this effect is weak and is not always observed in comparison with the main $\alpha$-process. It has been found that, $\alpha^{\prime}$-process is present in methyl ester form polymer. It represents the cooperative dipole motion of

$\mathrm{C}-\mathrm{O}-\mathrm{CF}_{2}-\mathrm{CF}-\left(\mathrm{CF}_{3}\right)$. For the present work, $\alpha^{\prime}$-relaxation can be assigned to the cooperative dipole motion of CPVC end groups [21] or the segmental motion of branching of CPVC. However, this effect in the present work is very weak for the stabilized CPVC sample. This may refer to the crooslinking formation between maleimide molecules and the $\pi$-bond of CPVC main chains. Crosslinking hinders the segmental motion of the branching of CPVC main chins. So, the $\alpha^{\prime}$ process has little chance to appear.

The third process, $\alpha$-peak, is obtained at $560 \mathrm{kHz}$. The peak position of this process shifts towards higher temperature with increasing frequency. This behavior results in the micro-Brownian motion between the polymeric main chains [5]. The peak height of $\alpha$-relaxation process for stabilized CPVC sample is higher than that obtained for pure component. Also, the shape of $\alpha$-peak for stabilized sample is broader than that for pure CPVC. This can be assigned to the diminution of the crystallinity of the stabilized CPVC sample, which is caused by the increase of the crosslinking density $[5,20]$. However, there is a competitive action of least three factors during the crosslinking:

1) Diminution of the existing physical network due to the hydrogen bonding;

2) Formation of a chemical network;

3) Crosslinking formation inside the amorphous regions.

Figures 3(a) and (b) show the temperature dependence of $\mathcal{E}^{\prime}$ for pure CPVC and that stabilized with $10 \mathrm{wt} \%$ of PM at some fixed frequencies. It is clear that, the values of $\varepsilon^{\prime}$ varies slowly with the increase in the temperature up to $T g \approx 363 \mathrm{~K}$. After $T g$, the magnitude of $\mathcal{E}^{\prime}$ increases gradually with further increasing in the temperature. Then, the dipoles of CPVC have sufficient energy, via heating, to orient themselves easily in the direction of the applied filed. So, the chain segments get sufficient thermal energy to speed up its rotational motion. This leads to increase in the polarization of the investigated sample [4,5]. Moreover, the increase of $\mathcal{E}^{\prime}$ with the temperature can be assigned to the disentanglement of the

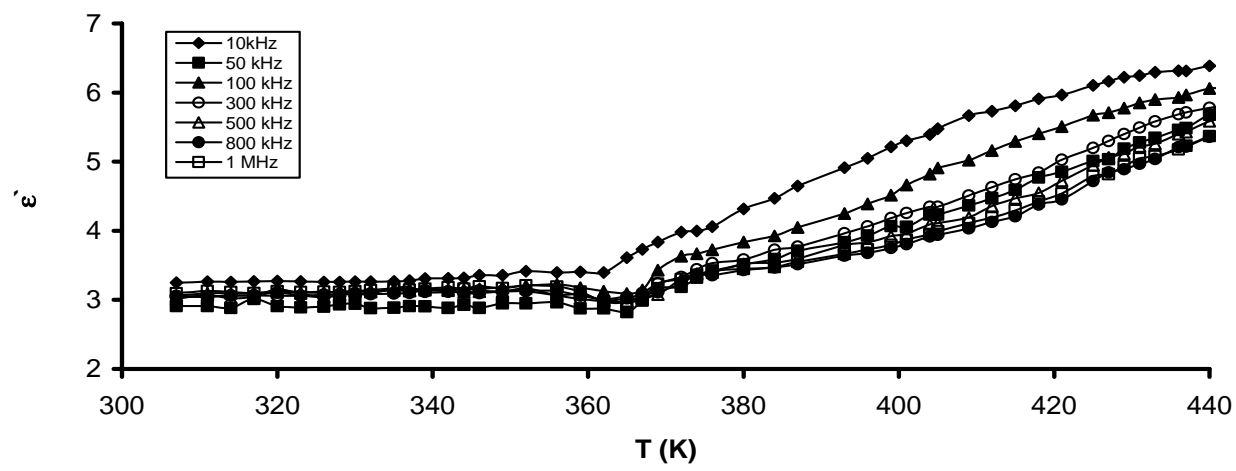

(a)

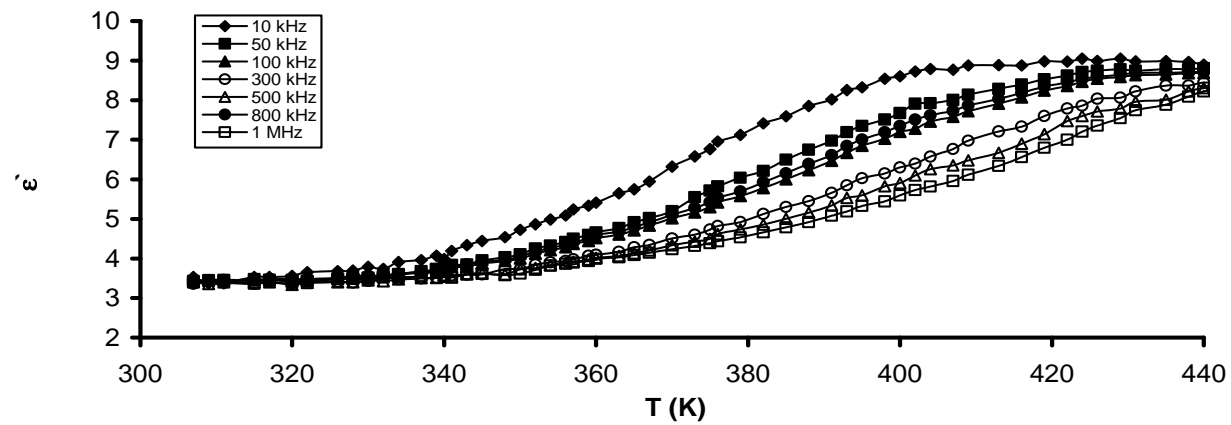

(b)

Figure 3. Temperature dependence of $\varepsilon^{\prime}$ (a) Pure CPVC and (b) CPVC stabilized with 10 wt\% of PM at some constant frequencies. 
molecular chains. It becomes easier due to the temperature induced molecular vibrations whereas at low temperature the chains are tightly packed and strongly held [8].

Figures 4(a) and (b) show the temperature dependence of $\varepsilon^{\prime \prime}$ for pure CPVC and that stabilized with 10 $\mathrm{wt} \%$ of PM at some fixed frequencies. It is observed that, $\mathcal{E}^{\prime \prime}$ for both pure and stabilized CPVC sample undergoes $\alpha$-relaxation process. The peak of this process for pure and stabilized CPVC sample was obtained at $405 \mathrm{~K}$ and $387 \mathrm{~K}$, at $10 \mathrm{kHz}$, respectively. Consequentially, the addition $10 \mathrm{wt} \%$ of PM to CPVC structure leads to decrease $T g$ of CPVC by about $27 \mathrm{~K}$. So, one can suggest that, PM molecules increase the free volume within the CPVC structure. In addition, the increment of the free volume lowers the chain-chain interaction within the backbone structure of CPVC. Then, PM molecules may screen the polar groups of CPVC and prevent the formation of polymer-polymer bond. Also, it indicates that PM molecules behave as a plasticizer for CPVC. The plasticization effect was observed earlier for the addition of Cadmium laurate [22], Gadolinium chloride $\mathrm{GdCl}_{3}$ [20] and bisphenol A corncobs [21] as stabilizers for PVC.

The temperature dependence of the maximum loss peak frequency $f_{\max }$ is displayed in Figure 5. The behavior of $f_{\max }$ can be described by Arrheniuss relation[23]:

$$
f_{\max }=f_{o} \exp \left(-\frac{E_{a}}{k T}\right)
$$

where $f_{o}$ is constant, $k$ is the Boltzmann's constant and $E_{a}$ is the activation energy. The calculated values of $E_{a}$ for pure and stabilized CPVC sample are 307 "kJ/mol" and 152 " $\mathrm{kJ} / \mathrm{mol}$ " respectively. The values of $E_{a}$ are disagreement with those reported in literature [4,20,24-26]. This may be assigned to the presence of the micro-inhomogeneities inside the investigated sample [14].

The temperature dependence of $\Delta \varepsilon$ for pure CPVC and that stabilized with $10 \mathrm{wt} \%$ of PM is displayed in Figure 6. It is seen that, $\Delta \varepsilon$ is proportional to the temperature. This can be attributed to the cooperation that exists between thermal energy and the electric field effects for dipole alignments. The increment of the thermal energy of the $\mathrm{C}-\mathrm{Cl}, \mathrm{C}=\mathrm{O}$, and phenyl groups will tend to enhance the alignment of themselves with the direction of the electric field and therefore the enhancement of $\Delta \varepsilon$ will expected.

\subsection{Electric Modulus Formalism}

The complex modulus, $M^{*}$, formulism is a very important and convenient tool to analyze and interpret the dielectric relaxation of polymeric material $[4,5]$. The main advantage of $M^{*}$ formulism is that the electrode polarization effect can be suppressed [27]. As the electrode effect is very important in the system, we have analyzed the dielectric spectrum by $M^{*}$ which can be evolved from the following relations [4.5]:

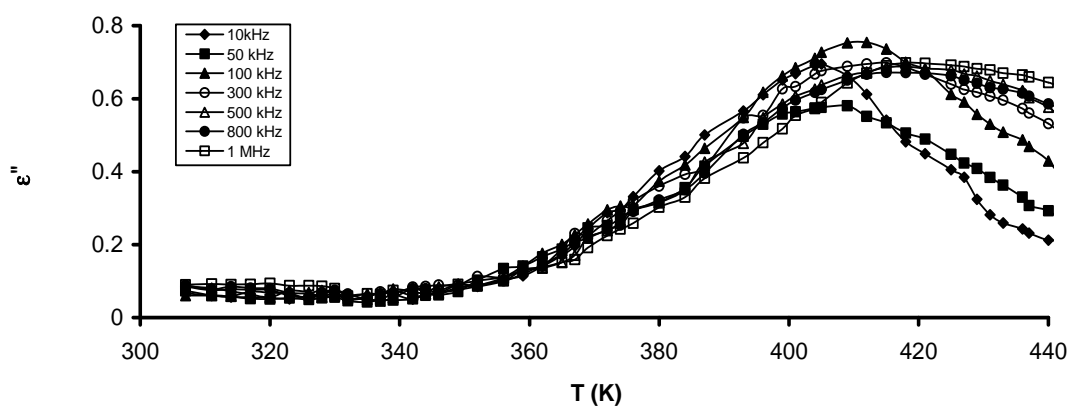

(a)

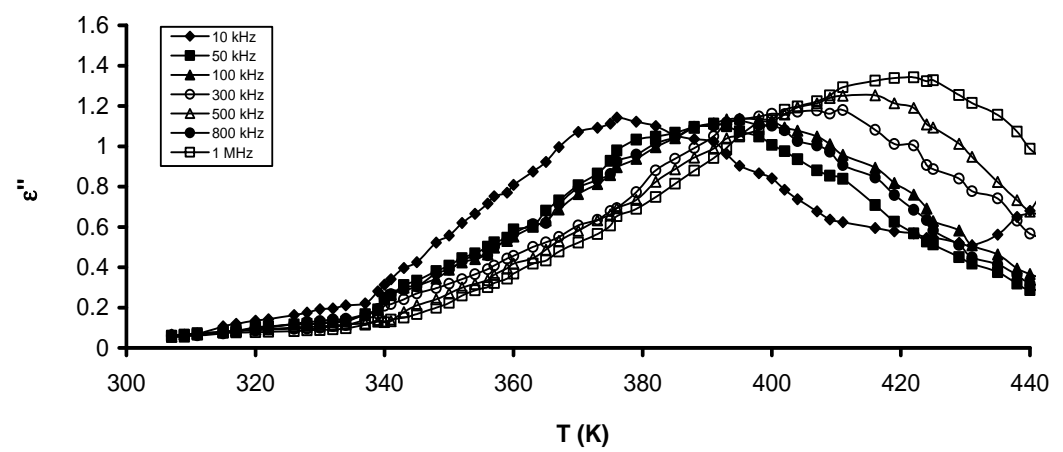

(b)

Figure 4. Temperature dependence of $\varepsilon^{\prime \prime}$ (a) Pure CPVC and (b) CPVC stabilized with 10 wt\% of PM at some constant frequencies. 


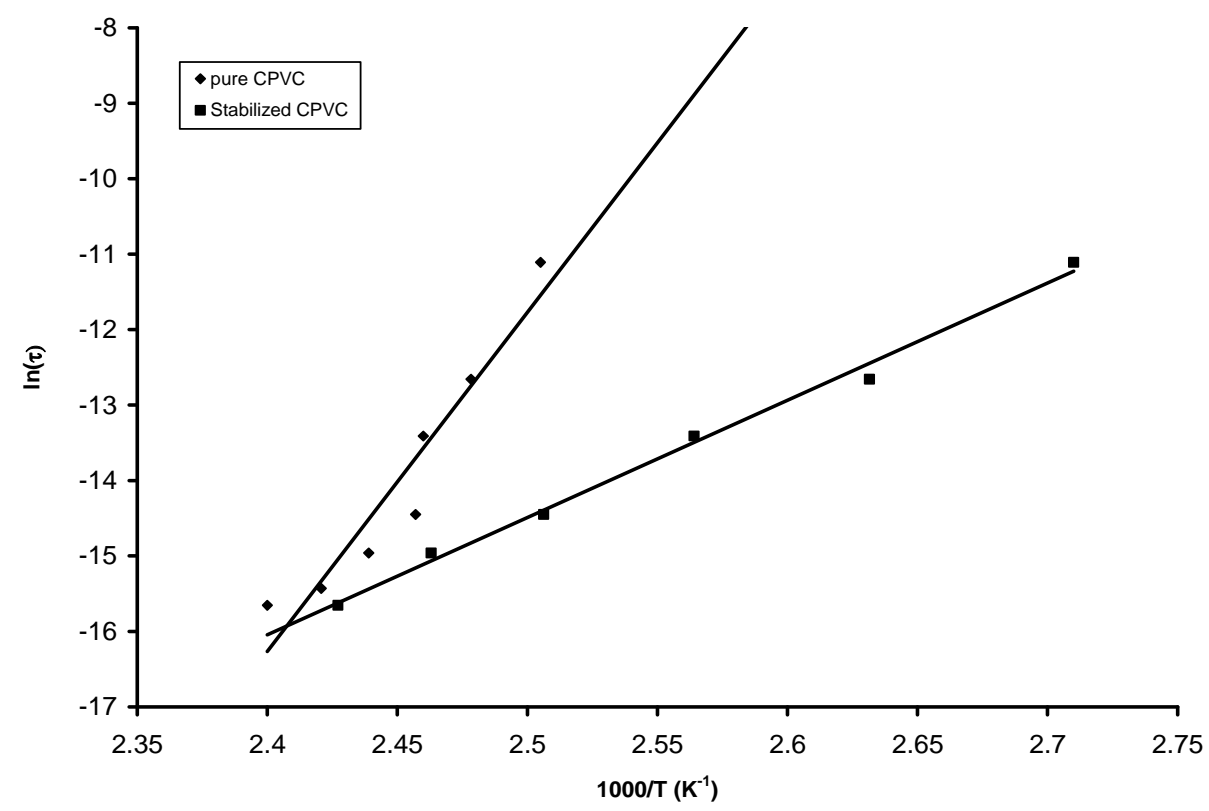

Figure 5. The relation between $\ln \left(\tau_{\sigma}\right)$ and $1000 / T$ for pure CPVC and that stabilized with 10 wt $\%$ of $P$.

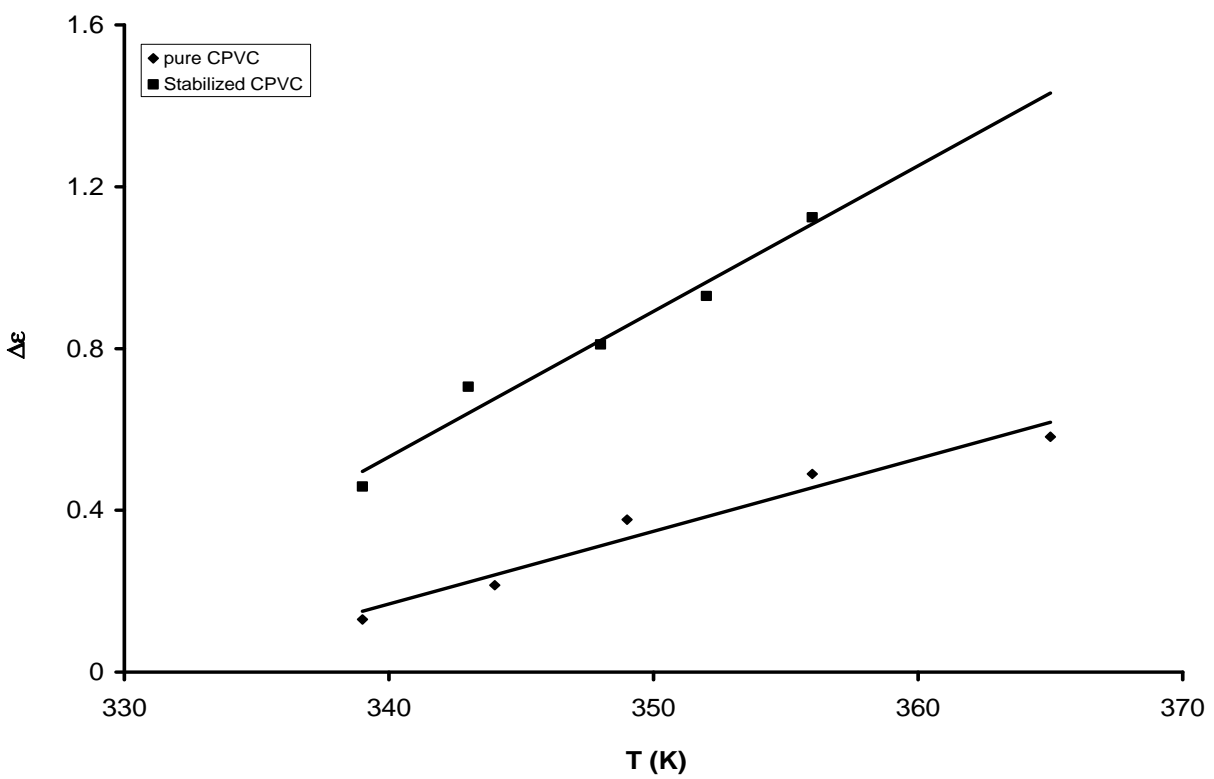

Figure 6. The temperature dependence of $\Delta \varepsilon$ for pure CPVC and that stabilized with 10 wt $\%$ of PM.

$$
\begin{aligned}
M^{\prime} & =\frac{\varepsilon^{\prime}}{\left(\mathcal{E}^{\prime 2}+\mathcal{E}^{\prime 2}\right)} \\
M^{\prime \prime} & =\frac{\varepsilon^{\prime \prime}}{\left({\left.\mathcal{E}^{\prime 2}+\mathcal{E}^{\prime 2}\right)}^{2}\right.}
\end{aligned}
$$

where $M^{\prime}$ and $M^{\prime \prime}$ are the real and the imaginary parts of the dielectric modulus, respectively. The frequency dependence of $M^{\prime}$ for pure CPVC and that stabilized with $10 \mathrm{wt} \%$ of PM at some fixed temperatures is shown in Figures 7(a) and (b). As the frequency increased $M^{\prime}$ of all CPVC samples is slightly increased and then reach to approximately constant value. The temperature dependence of $M^{\prime}$ for pure CPVC and that stabilized with 10 $\mathrm{wt} \%$ of PM at some fixed frequencies is shown in Figures 8(a) and (b). It is noticed that, at lower temperatures $M^{\prime}$ exhibits nearly constant value in the temperature ranges of $310-360 \mathrm{~K}$ and $310-330 \mathrm{~K}$ for pure and stabilized CPVC sample, respectively. This indicates that, the thermal stability of pure CPVC is higher than that obtained for the stabilized one. Moreover, the plasticization effect of PM molecules plays an important role for this process. At higher temperatures, $M^{\prime}$ for pure and stabilized CPVC samples tends to reach a constant value. This 


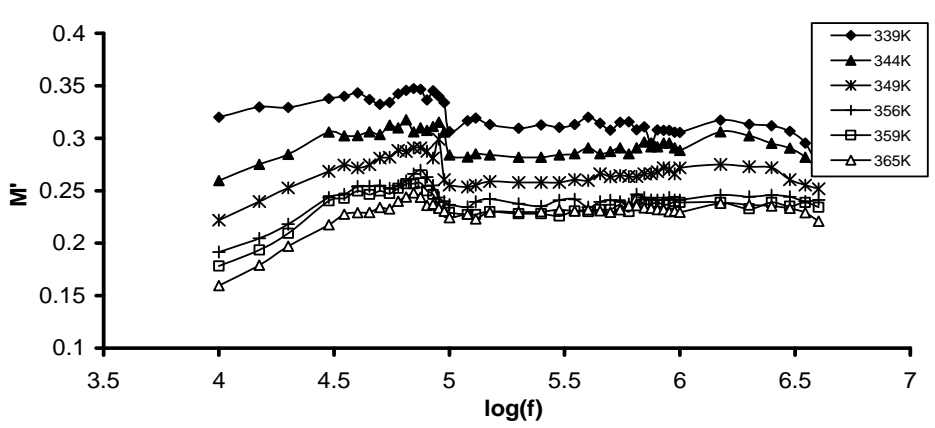

(a)

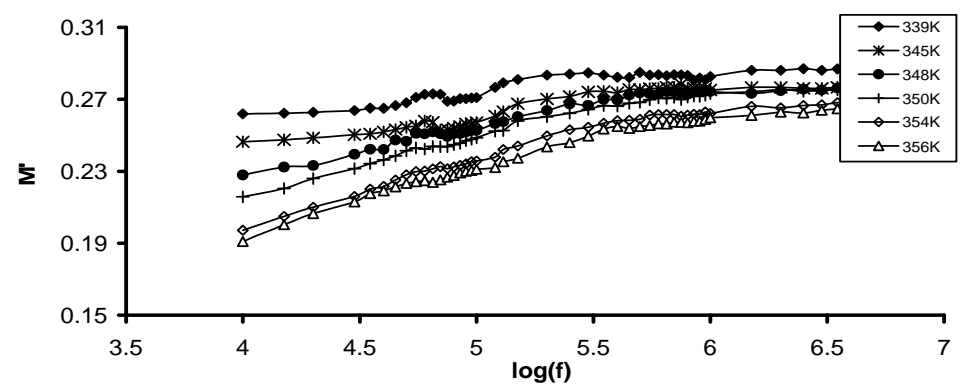

(b)

Figure 7. Frequency dependence of $M^{\prime}$ (a) Pure CPVC and (b) CPVC stabilized with 10 wt\% of PM at some constant temperatures.

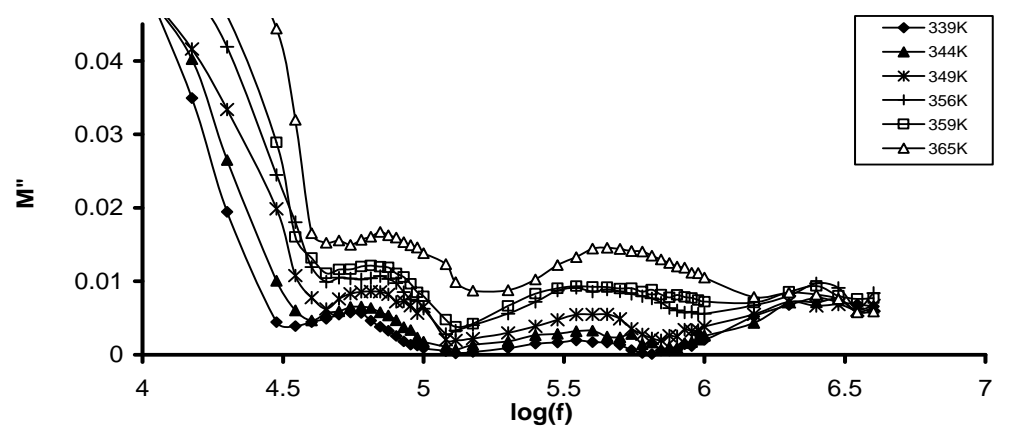

(a)

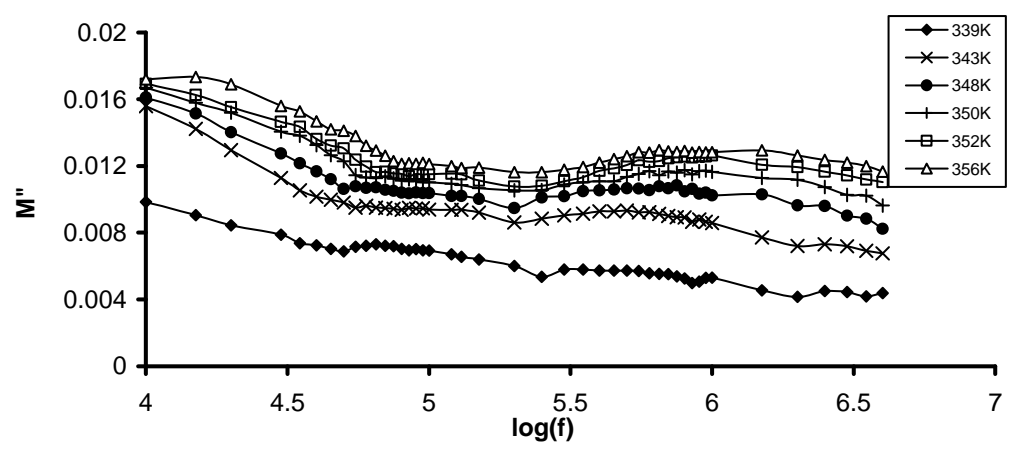

(b)

Figure 8. Frequency dependence of $M^{\prime \prime}$ (a) Pure CPVC and (b) CPVC stabilized with 10 wt \% of PM at some constant temperatures.

indicates thermally activated nature of the dielectric constant. In addition, the behavior of $M^{\prime}$ as a function of frequency, Figure 6, and as a function of temperature,
Figure 7, reveals that there is a role of electrode polarization for the dielectric relaxation.

Figures 9(a) and (b) illustrates the frequency dependence 


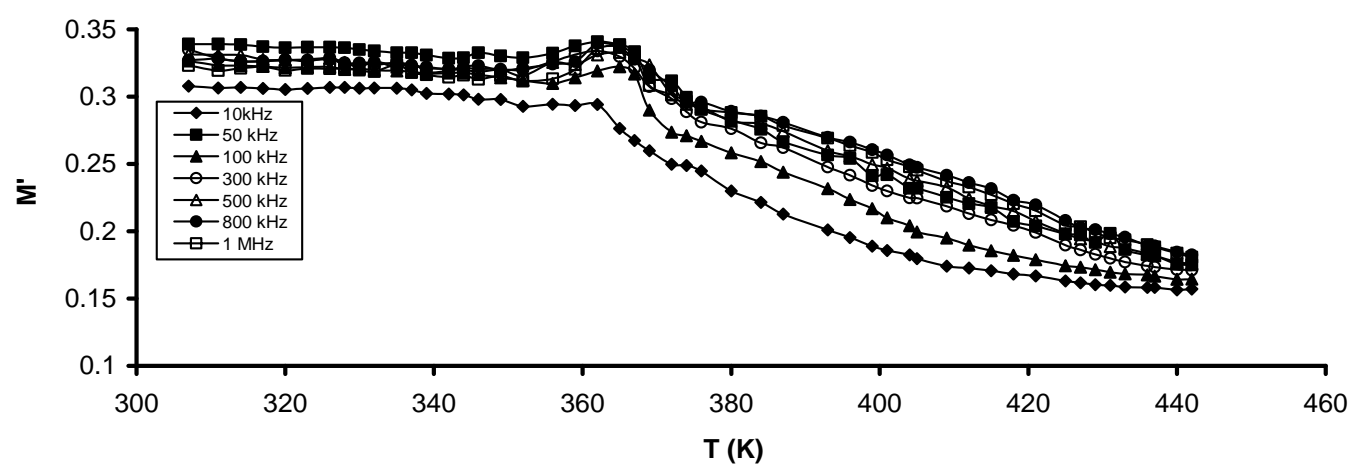

(a)

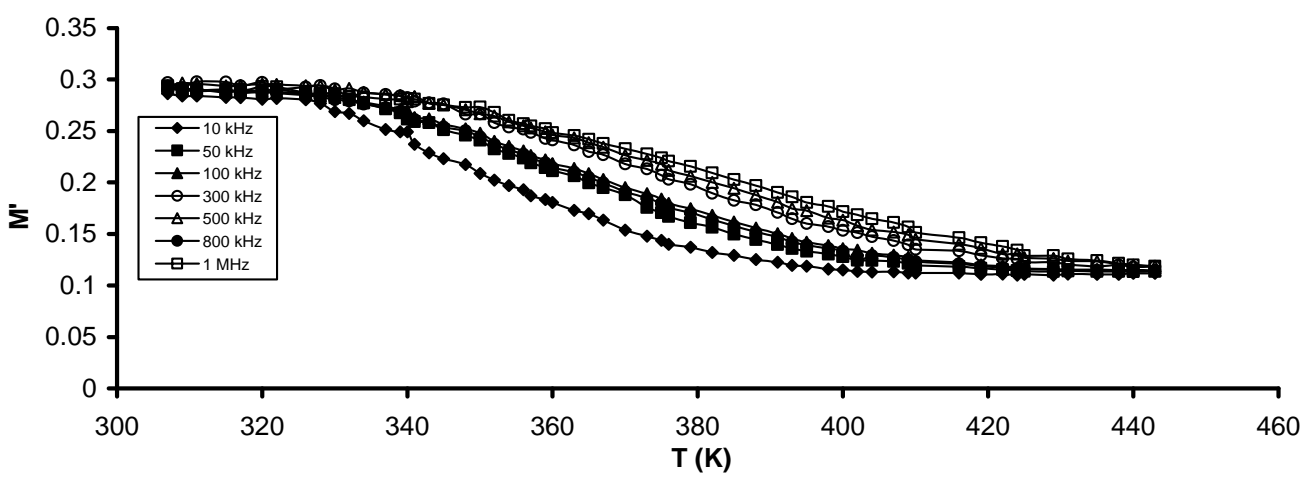

(b)

Figure 9. Temperature dependence of $M^{\prime}$ (a) Pure CPVC and (b) CPVC stabilized with 10 wt\% of PM at some constant frequencies.

of $M^{\prime \prime}$ for pure CPVC and that stabilized with $10 \mathrm{wt} \%$ of $\mathrm{PM}$ at some fixed temperatures. The behavior of $M^{\prime \prime}$ for all CPVC sample exhibits same trend that obtained for the frequency dependence of $\varepsilon^{\prime \prime}$, Figure 2. Three peaks $\rho$-, $\alpha^{\prime}$ - and the main $\alpha$-relaxation process were observed for pure CPVC at $20 \mathrm{kHz}, 63 \mathrm{kHz}$, and $400 \mathrm{kHz}$, respectively. Also, $M^{\prime \prime}$ of stabilized CPVC exhibits $\rho$-, $\alpha$-process at $25 \mathrm{kHz}$ and $1 \mathrm{MHz}$, respectively. This pattern provides information relating charge transport processes such as mechanism of electrical transport, conductivity relaxation, and ion dynamics as a function of temperature and frequency. The peak position of $\alpha$-process shifts toward the higher frequency side on increasing the temperature. The low-frequency side of the peak signifies the range of frequency in which ions can perform successful hopping from one site to the neighboring site. The highfrequency side of $M^{\prime \prime}$ peak represents the range of frequency in which the ions are spatially confined to their potential wells and the ions can make localized motion within the well. The region where the peak occurs is indicative of the transition from long-range to short-range mobility with increase in temperature. This type of behavior of the modulus spectrum is suggestive of a temperature-dependent hopping type mechanism for electrical conduction in the system [28]. The broadening of the peak points out the spread of relaxation with different mean time constants and non-Debye type of relaxation in CPVC.

The temperature dependence of $M^{\prime \prime}$ for pure and that stabilized CPVC sample at some fixed frequencies is shown in Figures 10(a) and (b). It is noticed that $M^{\prime \prime}$ for both pure and stabilized sample undergoes the main $\alpha$-relaxation process at $395 \mathrm{~K}$ and $365 \mathrm{~K}$ for pure and stabilized CPVC sample, respectively. This is due to the release of frozen-in all of the dipolar groups and their cooperative motion with adjoining segments of the main chains [29-31]. The relaxation time, $\tau$, has been calculating using the relaxation $\omega_{\max } \tau=1$ where $\omega_{\max }$ is the angular frequency at the peak of $M^{\prime \prime}$. The temperature dependence of $\tau$ for pure and stabilized CPVC is shown in Figure 11. The variation of $\tau$ with temperature follows the Arrhenius Equation [31]:

$$
\tau=\tau_{0} \exp \left(\frac{E_{a}}{k T}\right)
$$

where $\tau_{0}$ is constant. The calculated values of $E_{a}$ are 300 "kJ/mol" and 150 " $\mathrm{kJ} / \mathrm{mol}$ " for pure and stabilized CPVC sample, respectively.

On the other side the decrease of $T g$ for CPVC stabilized CPVC sample can be assign to dienopholic property of PM $[9,10]$. This property enables PM molecules to intervene within the conjugated CPVC system. This 


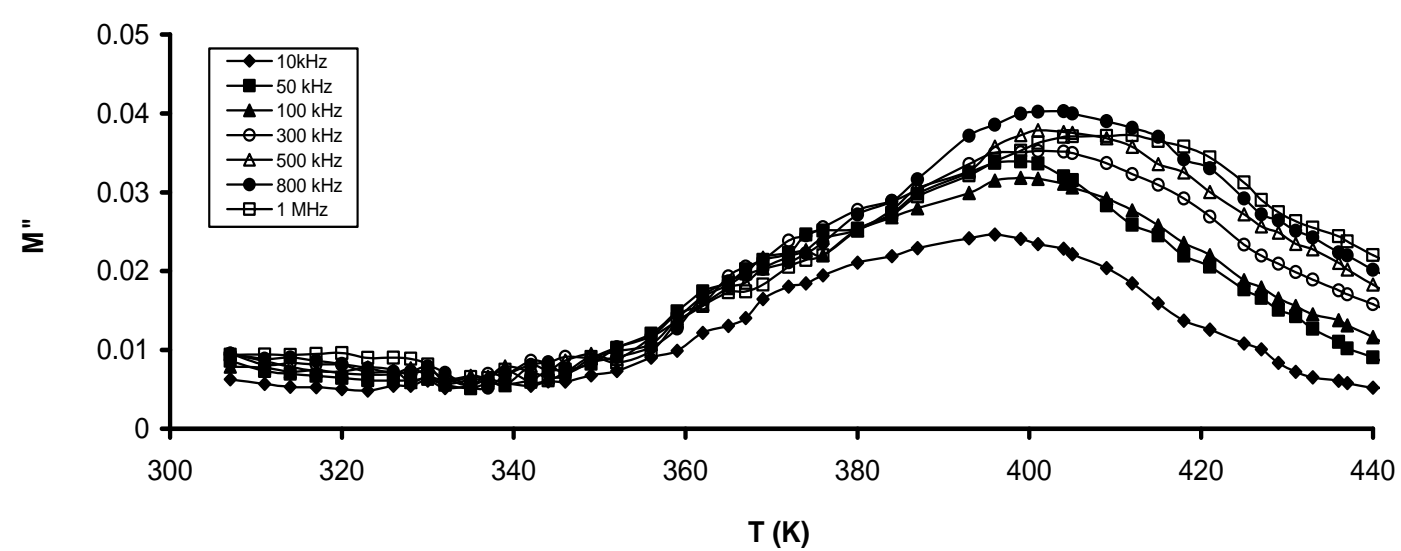

(a)

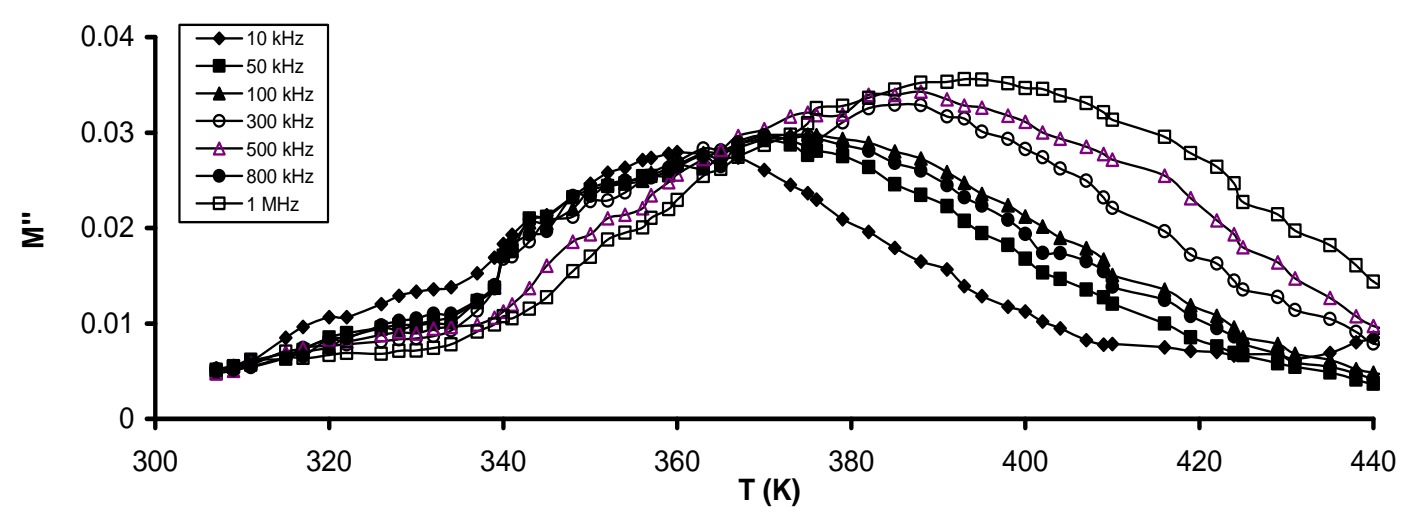

(b)

Figure 10. Temperature dependence of $M^{\prime \prime}$ (a) Pure CPVC and (b) CPVC stabilized with 10 wt\% of PM at some constant frequencies.

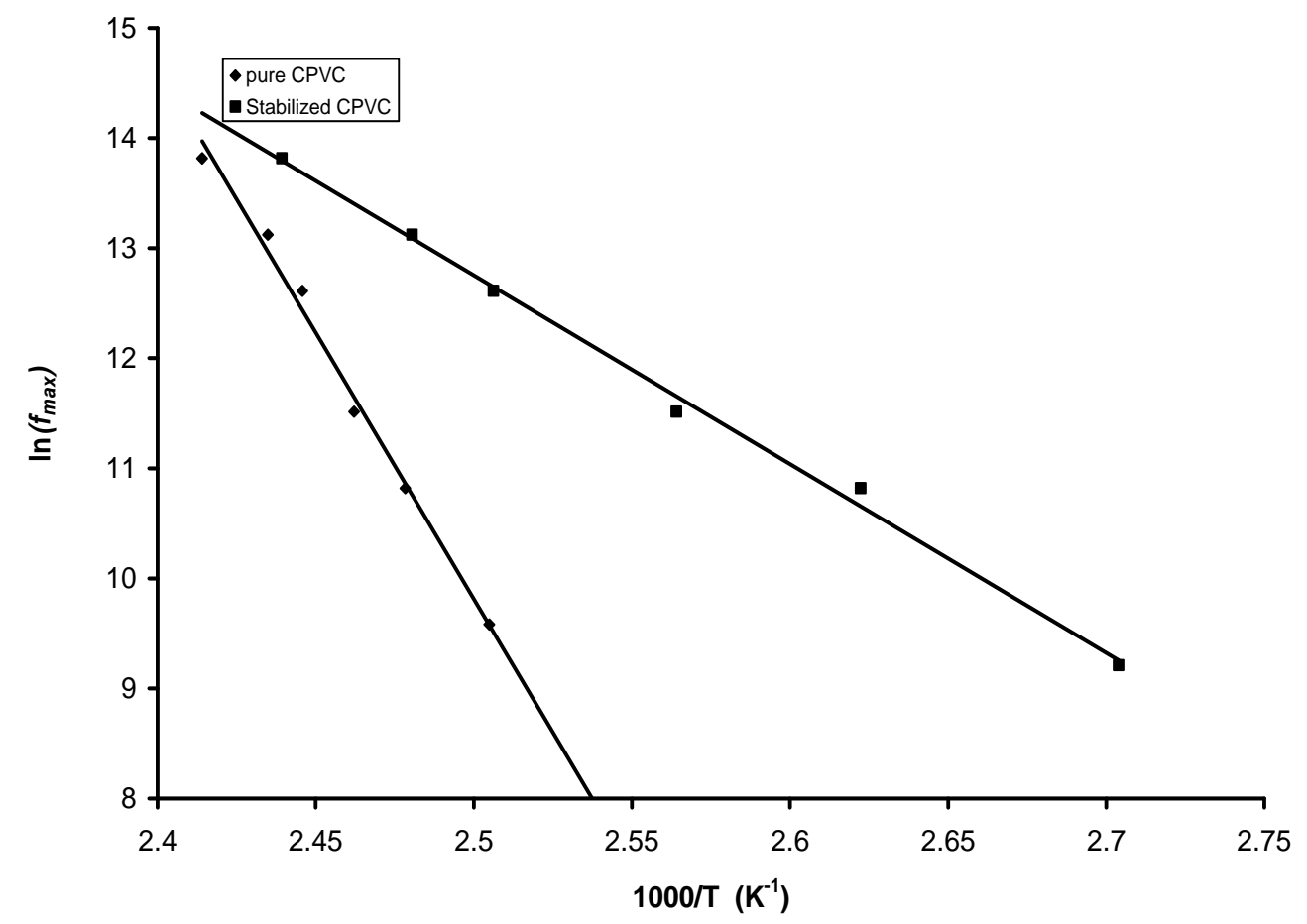

Figure 11. The relation between $f_{\max }$ and 1000/T for pure CPVC and that stabilized with 10 wt $\%$ of PM. 


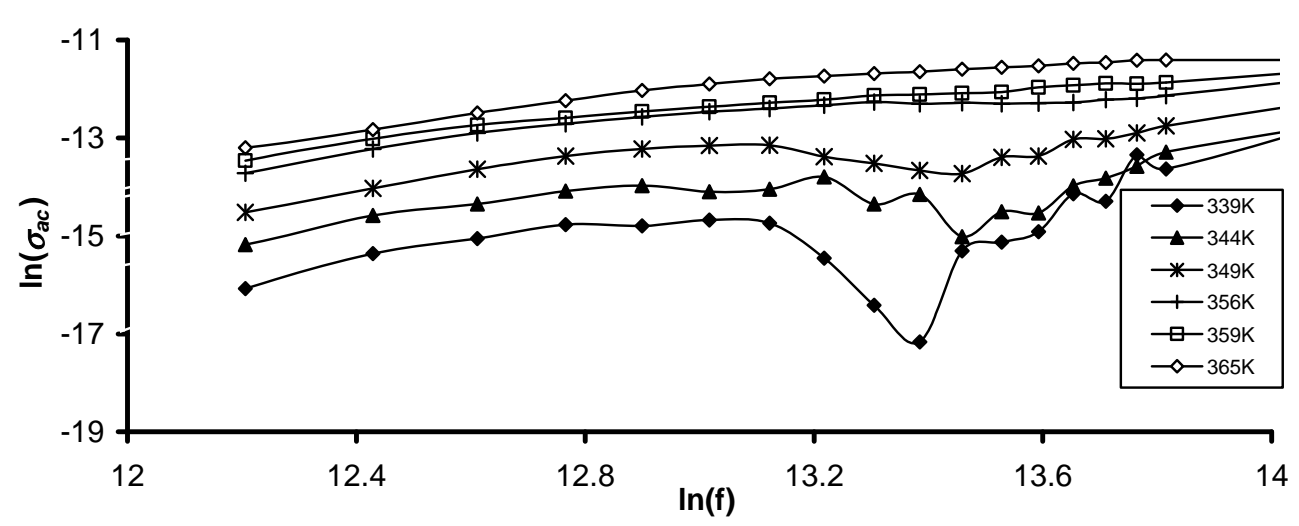

(a)

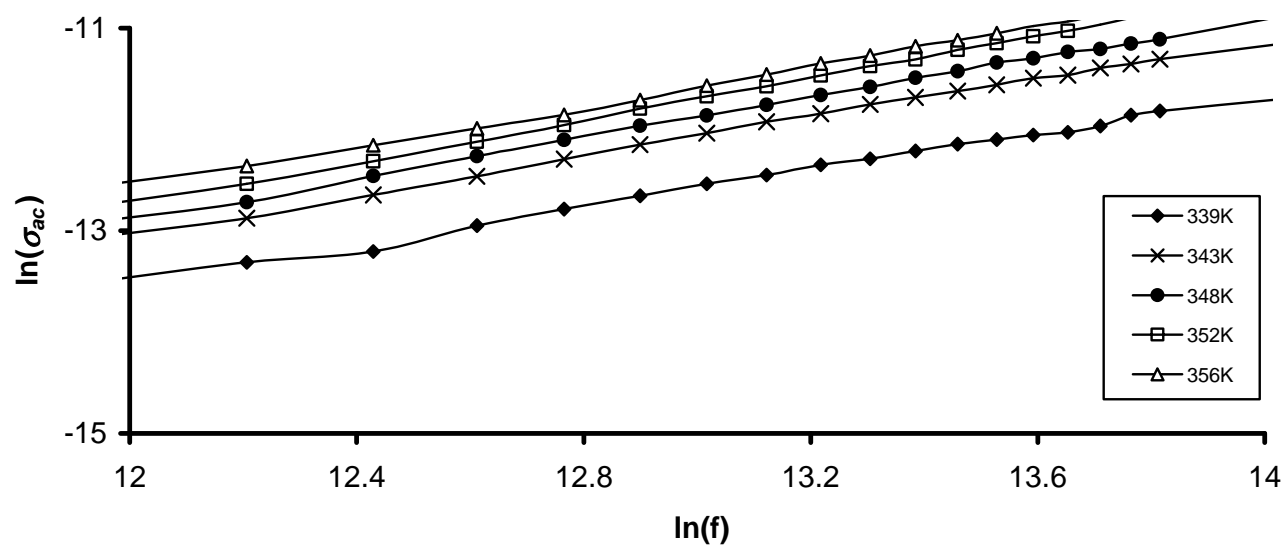

(b)

Figure 12. Temperature dependence of $\ln \left(\sigma_{\mathrm{ac}}\right)$ (a) Pure CPVC and (b) CPVC stabilized with 10 wt $\%$ of PM at some constant frequencies.

could be explained by the ability of maleimide molecules to react with the polyenes of CPVC structure to give a Diels-Alder type addition reaction $[9,10,32]$.

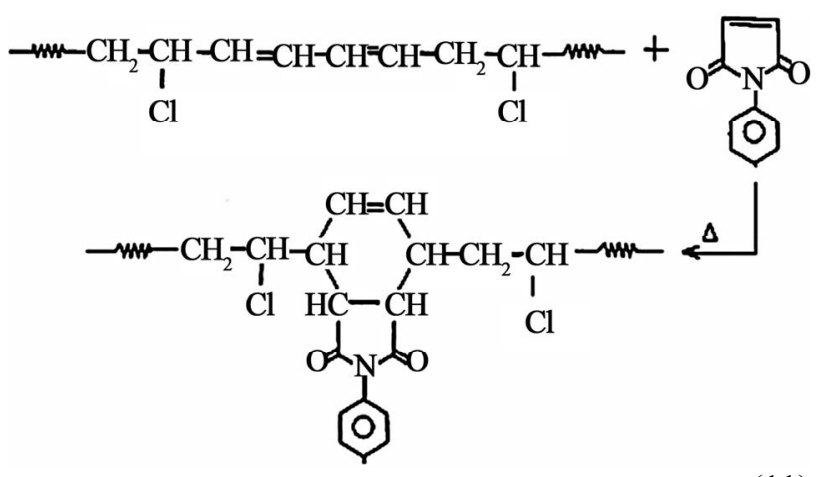

Not only Diels-Alder addition type reaction is responsible for the plasticization effect of PM molecules but also is responsible for the reduction of the number of the double bonds of CPVC structure. Consequently, PM molecules have a dual character. The first character is the stabilization effect due to the reducing of the double bonds ( $\pi$-bond) and the other is the plasticization effect due to the widely spacing between the main chains of the investigated sample. So, the reduction of $T g$ of CPVC in this case can be assign to weaken of the intermolecular interaction which makes the macromolecules take mainly flexible conformation.

\section{3. ac Conductivity}

The frequency dependence of the conductivity at constant temperature in the amorphous materials is given by [14]:

$$
\sigma(\omega)=\sigma(o)+A \omega^{s}
$$

where $\sigma(\omega)$ is the total conductivity, $\sigma(o)$ is the frequency independent conductivity, i.e. $\sigma_{\mathrm{dc}}$ and $A$ is constant. The exponent $s$ is a constant less than one. The frequency dependent or alternating current conductivity is given by $\sigma_{\mathrm{ac}}=A \omega^{s}$. Several theoretical models have been proposed to interpret the $\sigma_{\mathrm{ac}}$ for the amorphous semiconductors [20]. It is assumed that, the dielectric loss occurs because of the localized motion of charge carriers within a pair of sites [33]. Two distinct mechanisms have been proposed for relaxation phenomena quantum mechanical tunneling, QMT, of electrons or polarons through the barrier separating localized states and classical hopping over the same barrier. The frequency expo- 
nent $s$ in the QMT model can be described by [20]:

$$
s=1-\frac{4}{\ln (1 / \omega \tau)}
$$

This indicates that $s$ values are temperature independent. For the correlated barrier hopping, CBH, model [33], it was assumed that single electron motion is responsible. The frequency exponent $\mathrm{s}$ in the $\mathrm{CBH}$ model can be controlled by [34]:

$$
s=1-\frac{6 k T}{W_{H}}
$$

where $W_{H}$ is the maximum barrier height at infinite separation, which is called the polaron binding energy, i.e., the binding energy of the carrier in its localized sites. According Equation (13) $s$ exponent decreases with increasing temperature. To specify the dominant ac conduction mechanism for pure and stabilized CPVC sample, the frequency dependence of $\sigma_{\mathrm{ac}}$ at some fixed temperatures corresponding to the main $\alpha$-relaxation process has been considered. Figures 12(a) and (b) illustrates the frequency dependence of ac conductivity $\ln \left(\sigma_{\mathrm{ac}}\right)$ for pure CPVC and that stabilized with $10 \mathrm{wt} \%$ of PM at some fixed frequencies. It is clear that, $\sigma_{\mathrm{ac}}$ increases with increasing frequency and temperature. The calculated values of $s$ component for all CPVC samples are listed in Table 1. The data of s exponent reveal that the conduction mechanism for pure CPVC sample is governed by $\mathrm{CBH}$ while the predominant conduction mechanism for the stabilized CPVC sample could be QMT.

\section{Conclusion}

Addition of phenyl maleimide at $10 \mathrm{wt} \%$ to the CPVC causes a reduction of its $T g$ by about $27 \mathrm{~K}$, due to the plasticization effect of PM molecules. Dielectric losses and electric modulus behavior show three relaxation

Table 1. The values of the $s$ exponent for pure and stabilized CPVC samples.

\begin{tabular}{ccc}
\hline \multirow{2}{*}{ Temperature in K } & \multicolumn{2}{c}{$s$ exponent } \\
\cline { 2 - 3 } & Pure CPVC & Stabilized CPVC \\
\hline 339 & 0.95 & - \\
343 & - & 0.79 \\
344 & 0.93 & - \\
348 & - & 0.79 \\
349 & 0.86 & - \\
352 & - & 0.80 \\
356 & 0.82 & 0.81 \\
359 & 0.78 & - \\
365 & 0.74 & $\ldots \ldots$ \\
\hline
\end{tabular}

processes. The first process, $\rho$-relaxation, was assigned to MWS polarization. The second one, $\alpha^{\prime}$-process, was interpreted to the cooperation motion of the segmental motion of the branching of CPVC structure. The third one, $\alpha$-process, is due to the micro-Brownian motion of the main polymer chain. Finally, electric modulus and ac conductivity of the investigated samples reveal that the $\mathrm{CBH}$ is the most probable conduction mechanism for pure CPVC. While the conduction mechanism of the stabilized CPVC sample is QMT.

\section{Acknowledgements}

The author is very much thankful to Dr. Abir S. AbdelNaby, Fayoum University, for preparing phenyl maleimide.

\section{REFERENCES}

[1] E. O. Elakesh, T. Hull, D. Price and P. Carty, "Effect of Stabilisers and Lubricant on the Thermal Decomposition of Chlorinated Poly(Vinyl Chloride) (CPVC)," Polymer Degradation and Stability, Vol. 88, No. 1, 2005, pp. 4145. doi:10.1016/j.polymdegradstab.2004.04.027

[2] P. Carty, S. White, D. Price and L. Lu, "Smoke-Suppression in Plasticised Chlorinated Poly(Vinyl Chloride) (CPVC)," Polymer Degradation and Stability, Vol. 63, No. 3, 1999, pp. 465-468. doi:10.1016/S0141-3910(98)00075-5

[3] P. Carty and S. White, "The Effect of DOP Plasticizer on Smoke Formation in Poly(Vinyl Chloride)," Polymer, Vol. 33, No. 5, 1992, pp. 1110-1111. doi:10.1016/0032-3861(92)90033-S

[4] S. Mahrous, T. A. Hanfy and M. S. Sobhy, "Dielectric Relaxation of Chlorinated Polyvinyl Chloride (CPVC) Stabilized with Cyanoguanidine," Current Applied Physics, Vol. 7, No. 6, 2007, pp. 629-635. doi:10.1016/j.cap.2007.01.002

[5] S. Mahrous and T. A. Hanafy, "Dielectric Analysis of Chlorinated Polyvinyl Chloride Stabilized with Di- $n$-octyltin Maleate," Journal of Applied Polymer Science, Vol. 113, No. 1, 2009, pp. 316-320. doi:10.1002/app.29490

[6] N. Merah, F. Saghir, Z. Khan and A. Baz Aoune, "Effects on Fatigue Crack Growth Resistance of CPVC," Engineering Fracture Mechanics, Vol. 72, No. 11, 2005, pp 1691-1701. doi:10.1016/j.engfracmech.2004.12.002

[7] D. G. H. Ballard, A. N. Burgess, J. M. Dekoninck and E. A. Roberts, "The 'crystallinity' of PVC," Polymer, Vol. 28, 1987, pp. 3-9.

[8] R. D. Dworkin, "PVC Stabilizers of the Past, Present, and Future," Journal of Vinyl Technology, Vol. 11, No. 1, 1989, pp. 15-22. doi:10.1002/vnl.730110106

[9] S. B. Brown and C. M. Orlando, "Encyclopedia of Polymer Science and Engineering," Wiley, New York, 1988.

[10] M. W. Sabaa, N. A. Mohamed, E. H. Oraby and V. Yassin, "Organic Thermal Stabilizers for Rigid Poly(Vinyl Chloride) V. Benzimidazolylacetonitrile and Some of Its 
derivatives," Polymer Degradation and Stability, Vol. 76, No. 3, 2002, pp. 367-380. doi:10.1016/S0141-3910(02)00009-5

[11] A. S. Abdel-Naby and S. A. Nouh, "Stabilization of Poly(Vinyl Chloride) against Laser Radiation with Ethyl$N$-phenylmaleimide-4-caboxylate," Polymer Degradation and Stability, Vol. 76, No. 3, 2002, pp. 419-423. doi:10.1016/S0141-3910(02)00043-5

[12] M. Mujahid, D. S. Srivastava and D. K. Avasthi, "Dielectric Constant and Loss Factor Measurement of Polycarbonate, Makrofol KG Using Swift Heavy ion $\mathrm{O}^{5+}$," $R a$ diation Physics and Chemistry, Vol. 80, No. 4, 2011, pp 582-586. doi:10.1016/j.radphyschem.2010.12.007

[13] T. Sterzyński, J. Tomaszewska, K. Piszczek and K. Skórczewska, "The Influence of Carbon Nanotubes on the PVC Glass Transition Temperature," Composites Science and Technology, Vol. 70, No. 6, 2010, pp. 966-969.

[14] A. Yassin, M. Sabaa and N. Mohamed, " $N$-Substituted Maleimides as Photo-Stabilizers for Rigid Poly(Vinyl Chloride)," Polymer Degradation and Stability, Vol. 29, No. 3, 1990, pp. 291-303.

[15] A. Hassen, T. A. Hanafy, S. El-Sayed and A. Himanshu, "Dielectric Relaxation and Alternating Current Conductivity of Polyvinylidene Fluoride Doped with Lanthanum Chloride," Journal of Applied Physics, Vol. 110, No. 11, 2011, Article ID: 144119. doi:10.1063/1.3669396

[16] M. A. Ahmed, A. M. Basha, H. K. Marey and T. A. Hanafy, "Effect of Fast Neutrons and Radiation on Cobalt-Gelatin Film," Journal of Applied Polymer Science, Vol. 79, No. 10, 2001, pp. 1749-1755. doi:10.1002/1097-4628(20010307)79:10<1749::AID-AP P20>3.0.CO;2-H

[17] A. Chelkovski, "Dielectric Physics," Elsevier Science, Amsterdam, 1980.

[18] F. Kremer and M. Arntt, "Dielectric Spectroscopy of Polymeric Materials," American chemical Society, Washington DC, 1997.

[19] M. Narisawa and K. Ono, "Polymer Interaction and Structure of PVA-Cu(II) Complex: 1. Binding of a Hydrophobic Dye toward PVA-Cu(II) Complex," Polymer,Vol. 30, No. 8, 1989, pp. 1540-1545.

[20] T. A. Hanafy, "Dielectric Relaxation and AlternatingCurrent Conductivity of Gadolinium-Doped Poly(Vinyl Alcohol)," Journal of Applied Polymer Science, Vol. 108, 2008, pp. 2540-2549.

[21] M. A. Ahmed and T. A. Hanafy, "Dielectric Relaxation and Poole-Frenkel Conduction in Poly(Vinyl Chloride) Blends with Bisphenol A/Egyptian Corncob Resin," Journal of Applied Polymer Science, Vol. 109, No. 1, 2008, pp. 182-189. doi:10.1002/app.28075

[22] S. Mahrous, "Dielectric Analysis of the $\alpha$-Relaxation of PVC Stabilized with Cadmium Laurate," Polymer International, Vol. 40, No. 4, 1996, pp. 261-267.
[23] T. A. Hanafy, K. Elbana, S. Elsayed and A. Hassen, "Dielectric Relaxation Analysis of Biopolymer Poly(3-hydroxybutyrate)," Journal of Applied Polymer Science, Vol. 121, No. 6, 2011, pp. 3306-3313. doi:10.1002/app.33950

[24] R. Singh, J. Kumar, R. K. Singh, A. Kaur, R. D. P. Sinha and N. P. Gupta, "Low Frequency ac Conduction and Dielectric Relaxation Behavior of Solution Grown and Uniaxially Stretched Poly(Vinylidene Fluoride) Films," Polymer, Vol. 47, No. 16, 2006, pp. 5919-5928.

[25] M. Abdelaziz and M. M. Ghannam, "Influence of Titanium Chloride Addition on the Optical and Dielectric Properties of PVA Films," Physica B, Vol. 405, No. 3, 2010, pp. 958-964. doi:10.1016/j.physb.2009.10.030

[26] F. Bassiouni, F. Al-shamy, N. K. Madi and M. E. Kassem, "Temperature and Electric Field Effects on the Dielectric Dispersion of Modified Polyvinyl Chloride," Materials Letters, Vol. 57, No. 8-10, 2003, pp. 1595-1603.

[27] M. A. Ahmed, "Optical Absorption and Electrical Properties of Polyvinyl Alcohol (PVA)-Gelatin Blend," Indian Journal of Physics, Vol. 79, No. 10, 2005, pp. 1149-1155.

[28] G. M. Tsangaris, G. C. Psarras and N. Kouloumbi, "Evaluation of the Dielectric Behavior of Particulate Composites Consisting of a Polymeric Matrix and a Conductive Filler," Materials Science and Technology, Vol. 12, No. 7, 1996, pp. 533-538. doi: $10.1179 / 026708396790166037$

[29] C. N. Hampton, G. Carini, G. Dimarco and M. Lanza, "Temperature and Frequency Dependencies of the Complex Dielectric Constant of Poly(Ethylene Oxide) under Hydrostatic Pressure," Journal of Polymer Science Part B: Polymer Physics, Vol. 34, No. 3, 1996, pp. 425-433.

[30] G. S. Rillick and J. Runt, "A Dielectric Study of Poly(Ethylene-co-Vinylacetate)-Poly(Vinyl Chloride) Blends. I. Miscibility and Phase Behavior," Journal of Polymer Science Part B: Polymer Physics, Vol. 24, No. 2, 1986, pp. 279-302. doi:10.1002/polb.1986.090240206

[31] M. Ram and S. Chakrabarti, "Dielectric and Modulus Behavior of $\mathrm{LiFe}_{1 / 2} \mathrm{Ni}_{1 / 2} \mathrm{VO}_{4}$ Ceramics," Journal of Physics and Chemistry of Solids, Vol. 69, No. 4, 2008, pp. 905-912. doi:10.1016/j.jpcs.2007.10.008

[32] A. A. Yassin and M. W. Sabaa, "Degradation and Stabilization of Poly(Vinyl Chloride) JMS-REV," Macromolecular Chemistry and Physics C, Vol. 30, No. 3-4, 1990, pp. 491-558.

[33] T. A. Hanafy, "Transient and Steady State Currents of Bisphenol A Corncobs Sample," Advances in Materials Physics and Chemistry, Vol. 1, 2011, pp. 99-107.

[34] F. H. Elkader, W. H. Osman, K. H. Mahmoud and M. A. F. Basha, "Dielectric Investigations and ac Conductivity of Polyvinyl Alcohol Films Doped with Europium and Terbium Chloride," Physica B, Vol. 403, No. 19-20, 2008, pp. 3473-3484. 